\title{
HLA complex group 11 is involved in colorectal carcinoma cisplatin resistance via the $\mathrm{miR}-214-5 \mathrm{p} / \mathrm{SOX} 4$ axis
}

\author{
JIANPING XIE $^{1^{*}}$, JIAPING ZHU $^{2 *}, \mathrm{JIE} \mathrm{PANG}^{2}$ and YAPING MA ${ }^{2}$ \\ ${ }^{1}$ Department of Gastroenterology, The First Affiliated Hospital of Yangtze University, \\ The First People's Hospital of Jingzhou, Jingzhou, Hubei 434000; ${ }^{2}$ Department of Clinical Laboratory, \\ Affiliated Hospital of Hubei University of Arts and Science, Xiangyang Central Hospital, Xiangyang, Hubei 441000, P.R. China
}

Received August 26, 2020; Accepted February 8, 2021

DOI: $10.3892 / 01.2021 .12796$

\begin{abstract}
The aim of the present study was to investigate the roles and potential mechanisms of long non-coding RNA HLA complex group 11 (HCG11) in colorectal carcinoma. Reverse transcription-quantitative PCR was used to detect HCG11 expression in clinical tissues and survival analysis was performed to identify its prognostic value. In order to investigate its specific biological functions in colorectal carcinoma, the transfection technique was used for the knockdown and overexpression of HCG11. Dual-luciferase reporter gene and RNA pull-down assays were used to identify the binding association between HCG11 and microRNA (miR)-214-5p. Western blot analysis was used to detect the mechanism of epithelial-mesenchymal transition (EMT) regulation in tumor cells in the pathway downstream of HCG11. HCG11 level was high in colorectal carcinoma tissues, which was associated with poor patient prognosis; however, chemotherapy may prevent the upregulation of HCG11 in colorectal carcinoma. HCG11-knockdown suppressed the proliferation, migration and chemotherapeutic sensitivity of colorectal carcinoma cells, whereas HCG11-overexpression enhanced chemotherapeutic sensitivity. miR-214-5p was revealed to be a target gene, and upon direct interaction, a negative regulator of HCG11 in colorectal carcinoma cells. Inhibition of miR-214-5p reversed the restriction of HCG11 on the malignant activity of colorectal carcinoma cells, while miR-214-5p mediated the chemotherapy-related intracellular EMT pathway. In conclusion,
\end{abstract}

Correspondence to: Dr Yaping Ma or Dr Jie Pang, Department of Clinical Laboratory, Affiliated Hospital of Hubei University of Arts and Science, Xiangyang Central Hospital, 136 Jingzhou Street, Xiangcheng, Xiangyang, Hubei 441000, P.R. China

E-mail: zonhqian22@163.com

E-mail: xresn0@126.com

*Contributed equally

Key words: HLA complex group 11, colon carcinoma, chemotherapy resistance, microRNA-214-5p, epithelial-mesenchymal transition
HCG11 is a vital oncogene of colorectal carcinoma involved in mediating the chemotherapeutic resistance of tumors.

\section{Introduction}

Colorectal cancer (CRC) is the third most common malignant tumor worldwide, with more than one million newly diagnosed cases each year $(1,2)$. The 5-year survival rate of patients with CRC remains poor (3), albeit with a consecutive reduction in the morbidity and mortality rates of most cancer types, including colorectal carcinoma (4), in the past few decades. The major treatment strategies for CRC include surgery, radiotherapy and chemotherapy, and the primary reason for the poor prognosis of patients with $\mathrm{CRC}$ is the lack of reliable biomarkers and therapeutic targets (5). Therefore, it is of great significance to investigate the pathogenesis of CRC to improve diagnostic accuracy and treatment options.

Long non-coding RNAs (lncRNAs) are RNA molecules $>200$ nucleotides in length (6). IncRNAs were found to be important in tumorigenesis and to have various modes of action, such as chromatin modification, transcription and post-transcriptional regulation $(7,8)$. Emerging evidence indicates that lncRNAs may also play an important role in the diagnosis and treatment of CRC (9). IncRNA LINC00662 participates in the growth and metastasis of CRC by activating the ERK signaling pathway via the miR-340-5p/claudin-8/IL22 axis (10). IncRNA LINC00858 serves as an oncogene in $\mathrm{CRC}$, altering the expression of key genes hepatocyte nuclear factor $4 \alpha$ and WNK2 (11). Previous studies have also indicated that IncRNA HLA complex group 11 (HCG11) participates in the regulation of tumor progression in gastric cancer (12), brain glioma (13) and prostatic cancer (14), and is closely associated with the prognosis of patients with tumors. However, to the best of our knowledge, no study has investigated the expression profile of HCG11 and its role in CRC.

Epithelial-mesenchymal transition (EMT) is the acquisition of mesenchymal features, discovered and named by Greenburg and Hay in 1982 (15). EMT exerts its regulatory roles via complex molecular mechanisms, including epigenetics, posttranslational regulation and alternative splicing, as well as the influence of microRNAs (miRs) (16). In highly invasive human tumors, tumor cells possess significant characteristics of high malignancy for epithelial and stromal elements, and variations 
in EMT have been associated with poor prognosis $(17,18)$. In addition, increasing evidence indicates that EMT is also involved in tumor cell resistance to chemotherapy (19-21).

The present study investigated the expression profile and carcinogenesis role of HCG11 in CRC. Clinical data were collected to analyze its prognostic value, and tumor cells were cultured to perform functional experiments, including proliferation and migration assays. Furthermore, a chemotherapeutic drug resistance assay was performed by culturing resistant cell lines. Bioinformatics predictions and validation experiments were used to explore the mechanisms of HCG11 involved in CRC progression. Therefore, the present study investigated the roles of HCG11 in CRC to identify targets and biomarkers for the clinical diagnosis and treatment of this malignancy.

\section{Materials and methods}

Patients and specimens. A total of 23 pairs of CRC and paired non-cancerous tissues were collected at The First Affiliated Hospital of Yangtze University (Jingzhou, China) between June 2018 and December 2019. The mean age of patients (17 males and 6 females) was 61 years, ranging between 44 and 73 years. None of the patients received adjuvant treatment, including preoperative chemotherapy, prior to radical surgery. The present study was reviewed and approved by the medical ethics committee of The First Affiliated Hospital of Yangtze University, and all enrolled patients provided written informed consent. All experiments met the requirements of the Declaration of Helsinki. The collected tissue samples were frozen in liquid nitrogen and stored at $-80^{\circ} \mathrm{C}$ until use. All patients were followed up for long-term clinical data, including overall survival (OS) and tumor prognosis.

Reverse transcription-quantitative $(R T-q) P C R$. Total RNA was extracted from tissues and cells using TRIzol ${ }^{\circledR}$ reagent (Ambion; Thermo Fisher Scientific, Inc.). The corresponding cDNA was obtained by reverse transcription of miRNA, mRNA and lncRNA using the TaqMan miR kit (Applied Biosystems; Thermo Fisher Scientific, Inc.) or ImProm-II kit (Promega Corporation) according to the manufacturer's instructions, respectively; qPCR was conducted using SYBR Premix EX Taq ${ }^{\mathrm{TM}}$ (Takara Biotechnology Co., Ltd.) per the manufacturer's instructions $\left(95^{\circ} \mathrm{C}\right.$ for $30 \mathrm{sec}$, followed by 40 cycles at $95^{\circ} \mathrm{C}$ for $4 \mathrm{sec}$ and $60^{\circ} \mathrm{C}$ for $\left.34 \mathrm{sec}\right)$. Gene expression was quantified using the $2^{-\Delta \Delta \mathrm{Cq}}$ method (22) with U6 and GAPDH as a relative quantitative reference. The primer sequences were as follows: HCG11 forward, 5'-GCTCTATG CCATCCTGCTT-3' and reverse, 5'-TCCCATCTCCATCAACCC-3'; SOX4 forward, 5'-GCAAGATCATGGAGCAGTCG-3' and reverse, 5'-GGG CCGGTACTTGTAGTCG-3'; GAPDH forward, 5'-CTGGGC TACACTGAGCACC-3' and reverse, 5'-AAGTGG TCGTTGA GGGCAATG-3'; miR-214-5p forward, 5'-ACACTCCAGCTG GGACAGCAGGCACAGAC-3' and reverse, 5'-CTCAACTGG TGTCGTGGA-3'; and U6 forward, 5'-GCGCGTCGT GAAGCGTTC-3' and reverse, 5'-GTGCA GGGTCCGAGGT-3'.

Cell culture. Primary CRC cell lines (HCT116, SW620, SW480 and HT-29) and HIEC-6 normal colon tissue cells were purchased from the American Type Culture Collection. The cell lines were verified by STR profiling before experimental use. All cells were cultured using Dulbecco's modified Eagle's medium (Gibco; Thermo Fisher Scientific, Inc.) with $10 \%$ fetal bovine serum (Thermo Fisher Scientific, Inc.), and maintained at $37^{\circ} \mathrm{C}$ with $5 \% \mathrm{CO}_{2}$.

Transfection. Small interfering (si)RNAs against SOX4 and HCG11 (si-SOX4 and si-HCG11, respectively), and the pcDNA3.1 vector for HCG11 overexpression (oe-HCG11) were all purchased from Invitrogen (Thermo Fisher Scientific, Inc.), and miR-214-5p mimics, miR-214-5p inhibitor and the corresponding negative controls were all purchased from Shanghai GenePharma Co., Ltd. siRNAs $(30 \mathrm{nM})$ or plasmids $(1.5 \mu \mathrm{g} /$ well $)$ were transfected into cells at $37^{\circ} \mathrm{C}$ for $24 \mathrm{~h}$ using Lipofectamine $^{\circledR} 2000$ transfection reagent (Invitrogen; Thermo Fisher Scientific, Inc.) according to the manufacturer's protocol, and cells were harvested for RT-qPCR analysis $48 \mathrm{~h}$ post-transfection. The oligonucleotide sequences were as follows: siRNA SOX4, 5'-GGACAGACGAAGAGUUUAATT-3'; siRNA HCG11, 5'-UUCUCCGAACGUGUCACGUTT-3'; siRNA negative control, 5'-GCCAGAAUGUUCCUAUUUATT-3'; miR-214-5p mimics, 5'-GGCCTGGCTGGACAGAGTTG-3'; miR-214-5p inhibitor 5'-ACAGCAGGCACAGACAGGCAG-3'; miRNA mimics negative control, 5'-CAGUACUUUUGUG UAGUACAA-3'; and miRNA inhibitor negative control 5'-GUGAGAAGUACCACCGAGACAG-3'.

Cell Counting Kit 8 (CCK-8). Cells were seeded into a 96-well plate ( 6 wells per group) at a density of $\sim 1 \times 10^{4}$ cells/well. After transfection, $10 \mu \mathrm{l}$ CCK- 8 reagent was added at $0,24,48$ or $72 \mathrm{~h}$, followed by a $2-\mathrm{h}$ incubation at $37^{\circ} \mathrm{C}$. To determine cellular proliferation activity, a microplate reader (iMARK; Bio-Rad Laboratories, Inc.) was used to measure the absorbance of each well at $450 \mathrm{~nm}$. In addition, a drug sensitivity assay was conducted using CCK-8 reagent. Transfected cells were seeded into a 96-well plate for $24 \mathrm{~h}$, and then cisplatin (Beijing Solarbio Science \& Technology Co., Ltd.) was added to the medium at a concentration of $0,20,40,60,80$ or $100 \mu \mathrm{M}$. The absorbance at $450 \mathrm{~m}$ of the corresponding wells was measured after $72 \mathrm{~h}$.

Colony formation assay. Cells were seeded into a 6-well plate at a density of $\sim 600$ cells/well. After 2 weeks of stable culture, the cells were stained with crystal violet dye for $15 \mathrm{~min}$ at room temperature, and the resulting colonies were counted. The experiment was repeated three times.

Cell cycle analysis. Cells were collected and resuspended in DMEM with $10 \%$ FBS at a density of $\sim 2 \times 10^{5}$ cells $/ \mathrm{ml}$. After digestion with RNase A, the cells were fixed with $75 \%$ ethanol at $4^{\circ} \mathrm{C}$ overnight. The cells were then stained with propidium iodide (BD Biosciences) for $15 \mathrm{~min}$ in the dark, and cell cycle analysis was conducted using a flow cytometer (FACScan; BD Biosciences) and analyzed using the NovoExpress software v1.2 (ACEA Bioscience, Inc.; Agilent Technologies, Inc.).

5-Ethynyl-2'-deoxyuridine (EdU) staining. CRC cells were seeded into a 96-well plate at $1 \times 10^{4}$ cells/well for $48 \mathrm{~h}$. The cells were subsequently added to $500 \mu \mathrm{l}$ EdU reagent (25 $\mu \mathrm{M}$; Guangzhou RiboBio Co., Ltd.) and incubated for $2 \mathrm{~h}$, followed by $30 \mathrm{~min}$ of fixation in $4 \%$ paraformaldehyde at room temperature. The cells were then permeabilized with 
0.5\% Triton X-100 for $10 \mathrm{~min}$, followed by Apollo and DAPI staining of diagnostic nuclei in a dark environment for $30 \mathrm{~min}$ at room temperature. Finally, the stained cells were observed and counted under a fluorescence microscope (magnification, x200; Olympus Corporation).

Transwell assay. Cellular migration assays were performed using an 8-mm Transwell, 24-well plate (Corning Life Sciences). CRC cells were premixed at $\sim 400$ cells $/ \mu 1$ in serum-free media, and $100 \mu \mathrm{l}$ was added to the upper chamber of the Transwell insert; $700 \mu \mathrm{l}$ culture medium containing $10 \%$ fetal bovine serum was added to the lower chamber. After incubation at $37^{\circ} \mathrm{C}$ for $24 \mathrm{~h}$, a swab was used to remove the cells in the upper chamber. CRC cells that had migrated into the lower chamber were fixed with $4 \%$ paraformaldehyde at room temperature for $15 \mathrm{~min}$ and stained using crystal violet dye for $20 \mathrm{~min}$ at room temperature. An inverted microscope (magnification, x200; Olympus Corporation) was used to observe and count the migrated cells. Cells in a total of six fields of view were randomly selected for statistical analysis.

Bioinformatics analysis. The bioinformatics prediction websites starBase (http://starbase.sysu.edu.cn/), TargetScan (http://www.targetscan.org/) and miRDB (http://mirdb. org/index.html) were used to predict the target genes of HCG11 and miR-214-5p. Gene Expression Profiling Interactive Analysis (GEPIA) (23) is an interactive web for analyzing The Cancer Genome Atlas (TCGA) and Genotype-Tissue Expression projects online, and it was used to assess SOX4 expression in CRC and normal tissues.

RNA pull-down assay. The complementary biotin-labeled DNA probe for HCG11 was synthesized by Shanghai GenePharma Co., Ltd. The biotin-labeled probe was dissolved in buffer according the manufacturer's protocol, Dynabeads ${ }^{\mathrm{TM}}$ M-280 streptavidin (Invitrogen; Thermo Fisher Scientific, Inc.) was added, and the sample was incubated for $10 \mathrm{~min}$ at $24-26^{\circ} \mathrm{C}$. Then, the beads were placed into $\mathrm{CRC}$ cell lysis solution overnight at $4^{\circ} \mathrm{C}$, and RNA fragments that specifically bound to the beads were extracted using TRIzol ${ }^{\circledR}$ reagent. The biotin-labeled full-length HCG11 sequence was used to specifically pull down miRNAs for subsequent quantitative RT-qPCR analysis.

Dual-luciferase reporter gene assay. A dual-luciferase reporter gene assay was used to verify the binding association of HCG11 and SOX4 to miR-214-5p. The 3' untranslated region (UTR) containing HCG11 or SOX4, and sequence fragments of miR-214-5p predicted binding sites, were ligated into luciferase reporter plasmids and used to co-transfect CRC cells together with miR-214-5p mimics or miR-214-5p inhibitor using Lipofectamine ${ }^{\circledR} 2000$ transfection reagent (Invitrogen; Thermo Fisher Scientific, Inc.); the imported mutant sequence fragment was used as the reference group. To determine the gene binding relationship, the dual-luciferase reporter gene system (Promega Corporation) was used to measure luciferase activity. Luciferase activity was measured $24 \mathrm{~h}$ after transfection and the results were normalized to Renilla luciferase activity.

Western blot analysis. Cell lysis was performed using RIPA reagent (Thermo Fisher Scientific, Inc.) containing a protease inhibitor. The BCA method was used to determine protein concentration. For western blot analysis, $20 \mu \mathrm{g}$ protein/lane were separated by $10 \%$ SDS-PAGE and transferred onto a PVDF membrane. The membrane was blocked with 5\% skimmed milk at room temperature for $2 \mathrm{~h}$ before incubation with primary antibodies overnight at $4^{\circ} \mathrm{C}$. For specific determination of the expression of the targeted proteins, horseradish peroxidase-conjugated secondary antibodies (1:2,000; cat. no. 7076; Cell Signaling Technology, Inc.) were added and incubated at room temperature for $2 \mathrm{~h}$. ECL solution (Pierce; Thermo Fisher Scientific, Inc.) was used for visualization, and GAPDH (Cell Signaling Technology, Inc.) was used as the internal reference. The primary antibodies used in experiments were as follows: Vimentin (1:1,000; cat. no. 49636; Cell Signaling Technology, Inc.); E-cadherin (1:1,000; cat. no. 14472; Cell Signaling Technology, Inc.); GAPDH (1:1,000; cat. no. 51332; Cell Signaling Technology, Inc.); and SOX4 (1:500; cat. no. ab70598; Abcam).

Statistical analysis. GraphPad 7.0 software (GraphPad Software, Inc.) was used for statistical analysis and graphical presentation. The data are presented as the mean \pm standard deviation, and the relevant experiments were repeated at least three times. Independent-sample t-tests and paired-sample t-tests were used for the analysis of significant differences between two groups. Multi-group comparison analysis was performed using one-way ANOVA validated by Bonferroni's post-hoc test. Survival analysis of clinical prognostic data was performed using the log rank test, and correlation analysis was performed using Pearson's correlation coefficient. According to the median expression of HCG11 in 23 pairs of specimens, the OS data were grouped into high and low expression groups, and Kaplan-Meier analysis was used to assess survival. $\mathrm{P}<0.05$ was considered to indicate a statistically significant difference.

\section{Results}

Chemotherapy inhibits the expression of HCG11 in CRC. To determine the expression profiles of HCG11 in CRC, and its potential effects on chemotherapy resistance in CRC cells, HCG11 expression was compared in clinical samples and cell lines. RT-qPCR analysis revealed that HCG11 expression was notably upregulated in CRC tissues compared with normal tissues (Fig. 1A). CRC cell lines (HCT116, SW620, SW480 and HT-29), and a normal colon cell line (HIEC-6), were selected for HCG11 expression detection. The results showed that HCG11 expression was consistently higher in CRC cells than in normal colon cells (Fig. 1B). Furthermore, the prognostic clinical data of CRC patients were randomly collected and survival analysis was conducted to verify the correlation between HCG11 expression and survival. The results revealed that CRC patients that highly expressed HCG11 had considerably lower overall survival times than low-expression patients (Fig. 1C), suggesting the value of HCG11 as a clinical prognostic marker in CRC. Chemotherapy resistance is one of the key factors for the poor prognosis of CRC. To further investigate the influence of HCG11 on the chemotherapeutic resistance of CRC, the HT-29 and SW480 cell lines were used to assess the response to chemotherapy after 24 and $48 \mathrm{~h}$. Changes in HCG11 expression during chemotherapy were then analyzed via RT-qPCR in order to determine the association between chemotherapy and HCG11. The results 

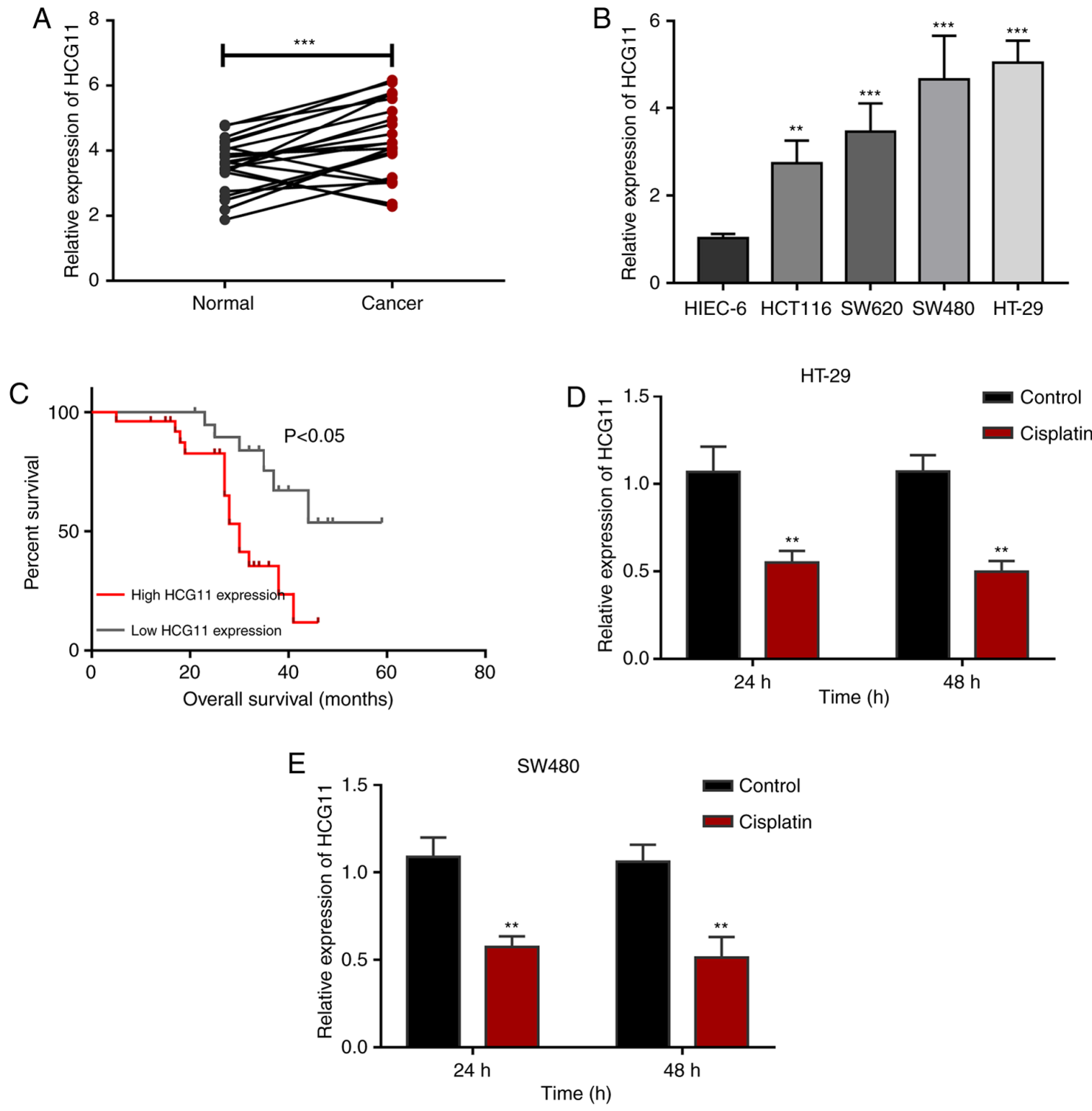

Figure 1. HCG11 expression in CRC. (A) RT-qPCR detection of an abnormal increase in HCG11 expression in CRC tissues. (B) Consistent increases in HCG11 expression in colorectal carcinoma cell lines, relative to normal colon cells, were detected by RT-qPCR. (C) CRC patients with high expression levels of HCG11 exhibited a poorer prognosis than patients with low expression. Downregulation of HCG11 expression in (D) HT-29 and (E) SW480 colorectal carcinoma cells 1-2 days after culture with $20 \mu \mathrm{M}$ cisplatin. ${ }^{* *} \mathrm{P}<0.01$ and $^{* * * *} \mathrm{P}<0.001$ vs. control. RT-q, reverse transcription-quantitative; HCG11, HLA complex group 11; CRC, colorectal cancer.

revealed that mean HCG11 expression in CRC cells was reduced following cisplatin treatment (Fig. 1D and E), suggesting that HCG11 expression was affected by tumor chemotherapy. In general, these findings indicate that HCG11 is highly expressed in CRC and is associated with poor prognosis. Additionally, chemotherapy had certain regulatory effects on HCG11 expression in CRC cells, implying that HCG11 may be involved in the resistance mechanisms of CRC to chemotherapy.

HCG11-knockdown suppresses the proliferation and migration of CRC cells. First, HCG11 expression was knocked down in CRC cells to identify its effects on cellular malignant phenotypes, including proliferation and migration; si-HCG11 transfection was conducted to knockdown HCG11 expression in HT-29 and SW480 cells. transfection efficiency was verified by RT-qPCR, which showed that HCG11 expression was notably suppressed in HT-29 and SW480 cells (Fig. 2A). Furthermore, the proliferation of
HT-29 and SW480 cells was assessed using the CCK-8 assay $24 \mathrm{~h}$ post-transfection. The results showed that the proliferation of CRC cells was significantly attenuated after HCG11-knockdown (Fig. 2B and C). Furthermore, EdU staining was performed to confirm the effects of HCG11-knockdown on CRC cell proliferation, which showed that the ratio of EdU-positive cells was markedly decreased, indicating that proliferation was attenuated (Fig. 2D). A colony formation assay was also performed to detect changes in cell colony formation after HCG11-knockdown. Following 2 weeks of culture, colony formation ability was also markedly suppressed, and the number of colonies formed was significantly reduced (Fig. 2E). Cellular migration was then evaluated using a Transwell assay, revealing that HT-29 and SW480 cell migration was significantly weakened following HCG11-knockdown. The number of migrated cells was considerably reduced compared with that of the control group (Fig. 2F and G). 
A

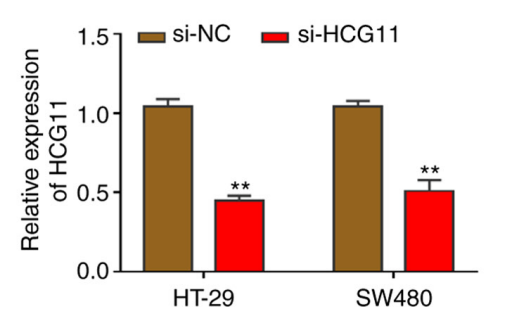

D

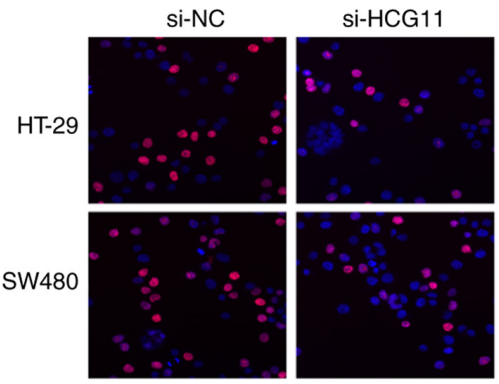

E

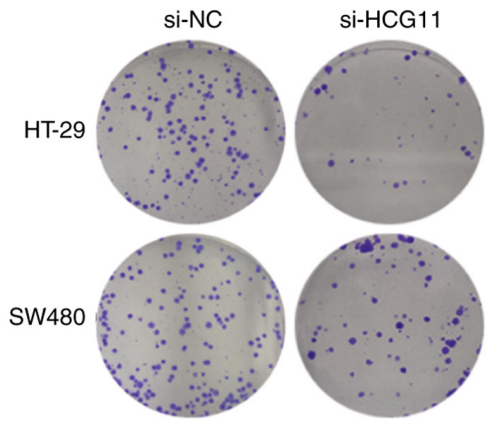

B
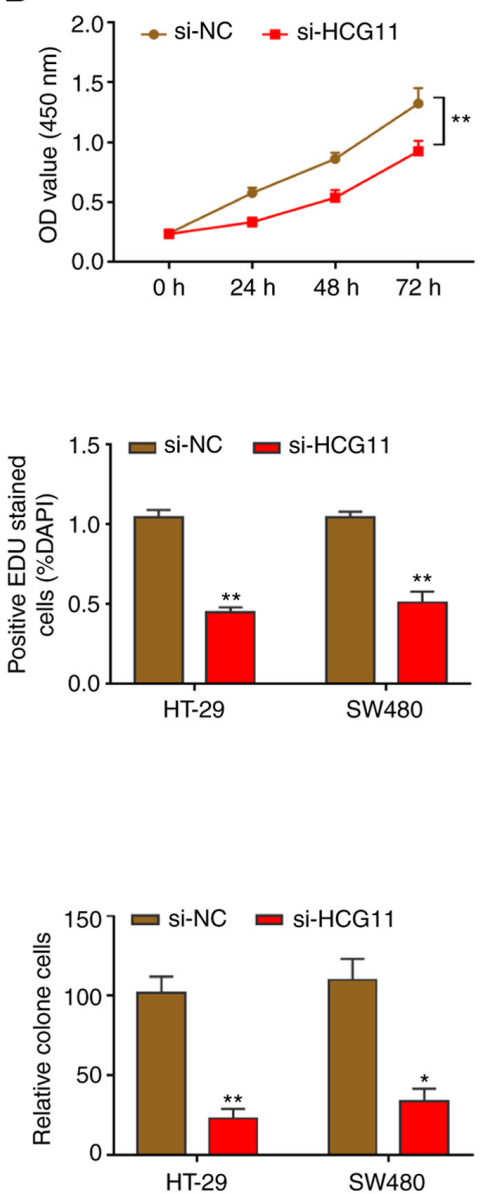

C

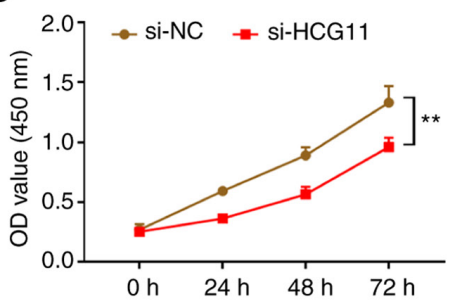

F
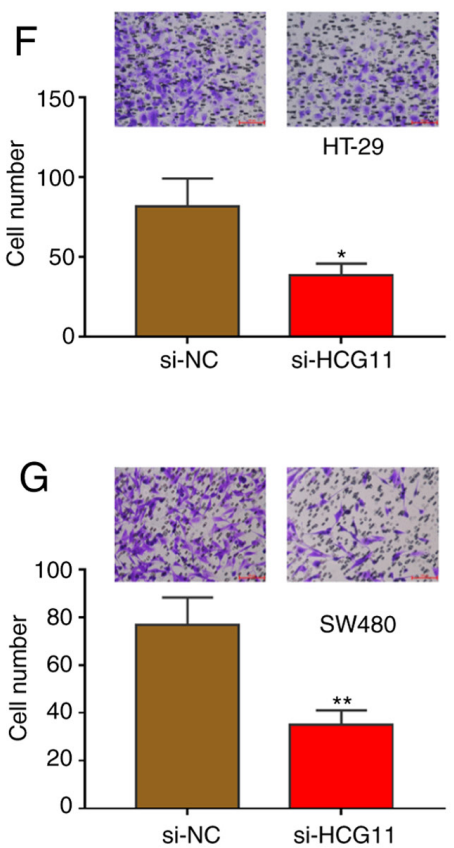

$\mathrm{H}$

$H$
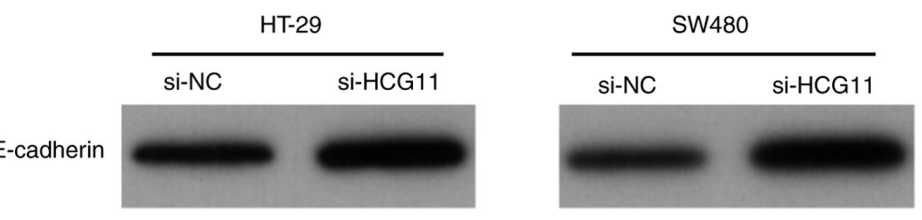

Vimentin

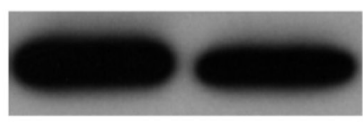

GAPDH
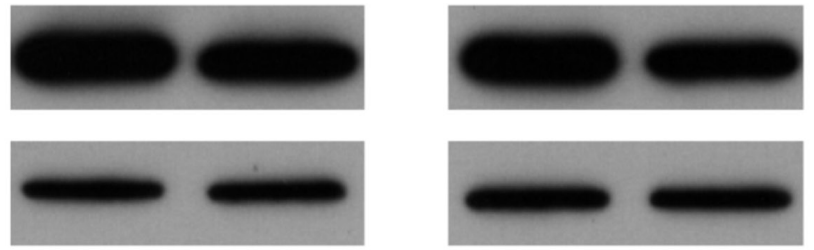

Figure 2. HCG11-knockdown suppresses the proliferation and migration of CRC cells. (A) Transfection of si-HCG11 notably downregulated HCG11 expression in colorectal carcinoma cell lines. Effects of HCG11-knockdown on the proliferation of (B) HT-29 and (C) SW480 colorectal carcinoma cells was assessed using the Cell Counting Kit-8. (D) EdU was used to determine the effects of HCG11-knockdown on the proliferation of colorectal carcinoma cell lines. (E) Effects of HCG11-knockdown on the clonal propagation of colorectal carcinoma cell lines in plate colony assay. Effects of HCG11-knockdown on the migration of (F) HT-29 and (G) SW480 colorectal carcinoma cell lines was assessed using Transwell assays. (H) HCG11-knockdown suppressed vimentin, but increased E-cadherin protein expression. $\mathrm{P}<0.05$ and ${ }^{* *} \mathrm{P}<0.01$ vs. control. Si, small interfering (RNA); NC, negative control; HCG11, HLA complex group 11; EdU, 5-ethynyl-2'-deoxyuridine.

EMT is the key mechanism mediating cellular proliferation and migration; therefore, the expression of EMT pathway-related proteins, such as E-cadherin and vimentin, was determined. As shown in Fig. 2H, HCG11-knockdown suppressed vimentin, but increased E-cadherin protein levels, reflecting the inhibition of the intracellular EMT pathway. These experimental results further demonstrate that HCG11-downregulation suppressed the proliferation and migration of CRC cells via the EMT pathway.
HCG11 induces chemotherapeutic resistance of CRC cells to cisplatin. Based on the aforementioned findings, the influence of HCG11 on the chemotherapeutic resistance of tumor cells was then investigated. RT-qPCR demonstrated that oe-HCG11 transfection resulted in HCG11-overexpression in HT-29 and SW480 cells (Fig. 3A). The effects of HCG11 on tumor cell sensitivity to chemotherapy were then investigated. Cell viability was detected $72 \mathrm{~h}$ post-cisplatin treatment, and HCG11-overexpression was found to restrain chemotherapeutic 
A

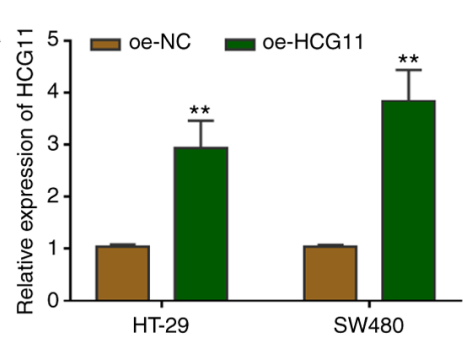

D
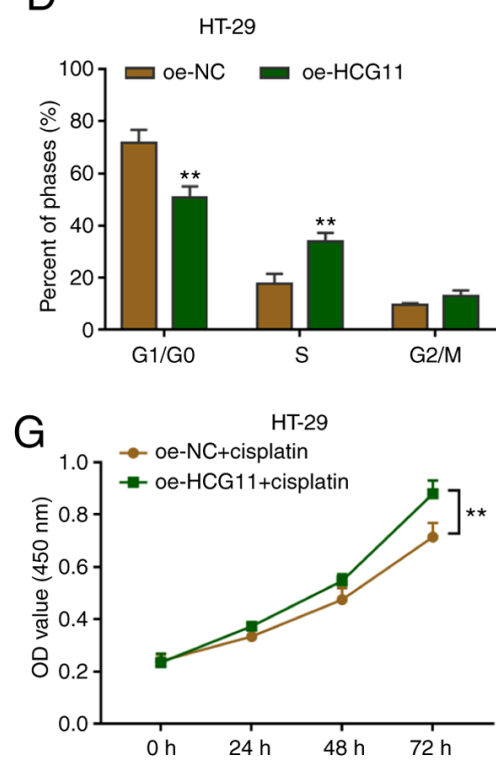

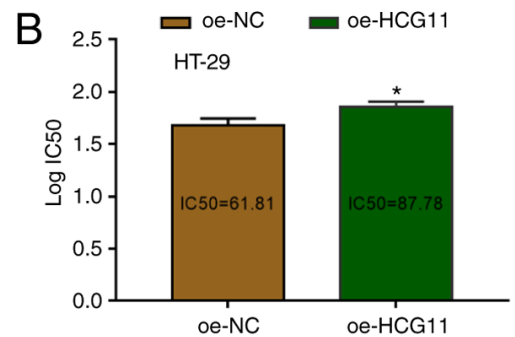

$\mathrm{E}$
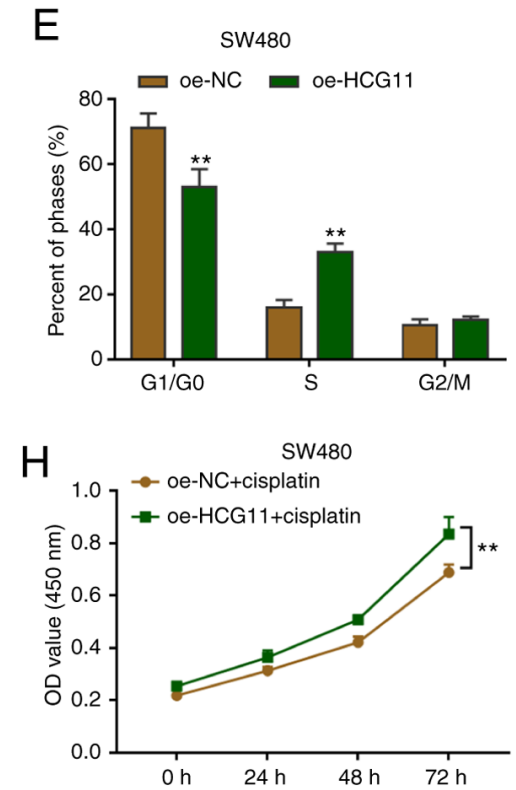

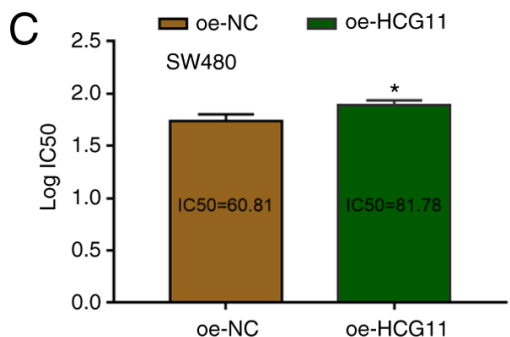

$\mathrm{F}$
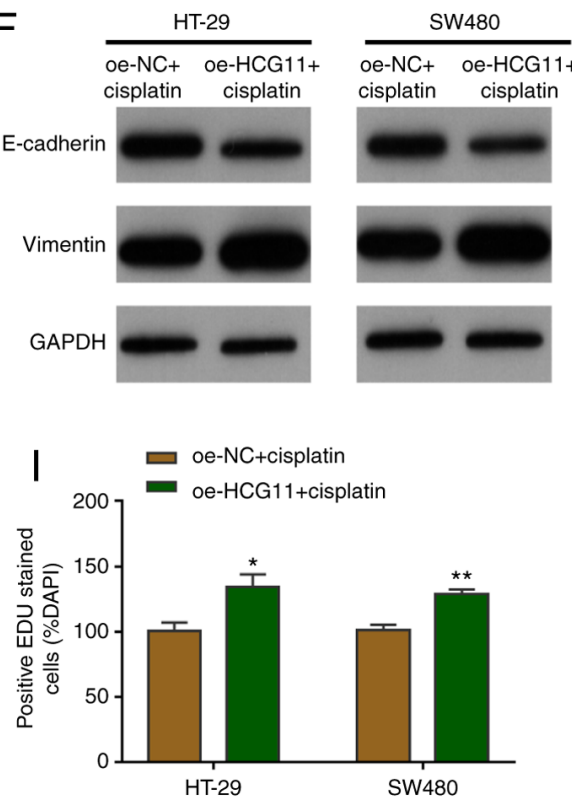
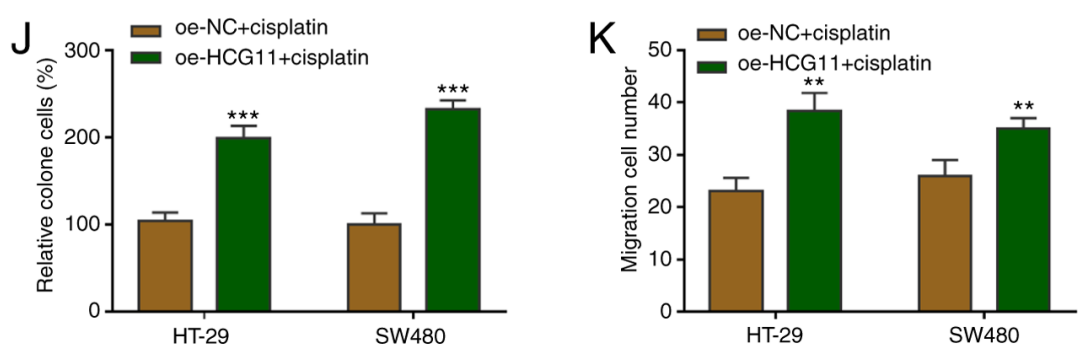

Figure 3. HCG11 induces chemotherapeutic resistance of CRC cells to cisplatin. (A) Transfection of oe-HCG11 notably upregulated HCG11 expression in colorectal carcinoma cell lines. (B) HT-29 and (C) SW480 colorectal carcinoma cells transfected with oe-HCG11 showed stronger chemotherapeutic resistance with higher $\mathrm{IC}_{50}$ values than the control groups. Overexpression of HCG11 decreased $\mathrm{G}_{1} / \mathrm{S}$ arrest in (D) HT-29 and (E) SW480 cells compared with the control cells following cisplatin treatment. (F). HCG11-overexpression reactivated the epithelial-mesenchymal transition pathway in colorectal cells following cisplatin chemotherapy. Overexpression of HCG11 enhanced the chemotherapeutic resistance of (G) HT-29 and (H) SW480 colorectal carcinoma cells, as shown using the Cell Counting Kit-8. (I) EdU and (J) colony formation assays showed that HCG11-overexpression enhanced the chemotherapeutic resistance of colorectal carcinoma cell lines. (K) Overexpression of HCG11 reduced the effects of chemotherapy on the migration of colorectal carcinoma cell lines, as shown using Transwell assays. ${ }^{*} \mathrm{P}<0.05,{ }^{* *} \mathrm{P}<0.01$ and ${ }^{* * *} \mathrm{P}<0.001$ vs. control. Oe, overexpression; HCG11, HLA complex group 11 ; EdU, 5-ethynyl-2'-deoxyuridine.

sensitivity and increase the IC50 value (Fig. 3B and C). A cell cycle assay was then performed to identify the cell death pattern during chemotherapy. The results showed that the overexpression of $\mathrm{HCG} 11$ decreased $\mathrm{G}_{1} / \mathrm{S}$ arrest compared with the control following cisplatin chemotherapy $(20 \mu \mathrm{M})$ (Figs. 3D and E, and S1A). Furthermore, the EMT pathway was significantly inactivated by consistent cisplatin treatment. However, HCG11-overexpression could reactivate the EMT pathway in CRC cells (Fig. 3F). In addition, the degree of chemotherapeutic tolerance was detected by determining cell viability, proliferation and migration abilities. CRC cells overexpressing HCG11 exhibited increased proliferative activity under culture with chemotherapy (Fig. 3G and H). Consistent with these findings, cellular EdU staining post-HCG11 overexpression showed that the proportion of EdU-positive cells was increased, suggesting stronger cellular proliferation activity (Figs. 3I and S1B). Cell clone formation experiments revealed stronger cisplatin resistance in CRC cells under treatment with chemotherapy drugs, and a higher growth rate of cell clones (Fig. 3J and S1C). Similarly, cellular migration experiments indicated that migration was enhanced relative to the control group, and that CRC cells overexpressing HCG11 resisted chemotherapeutic suppression (Fig. 3K and S1D). Collectively, the HCG11-overexpression experiments confirmed the effects of chemotherapeutic resistance on CRC cells, which was induced by the EMT pathway.

miR-214-5p is a target gene of HCG11 in CRC. As predicted by starBase, miR-214-5p was confirmed to be a target gene 

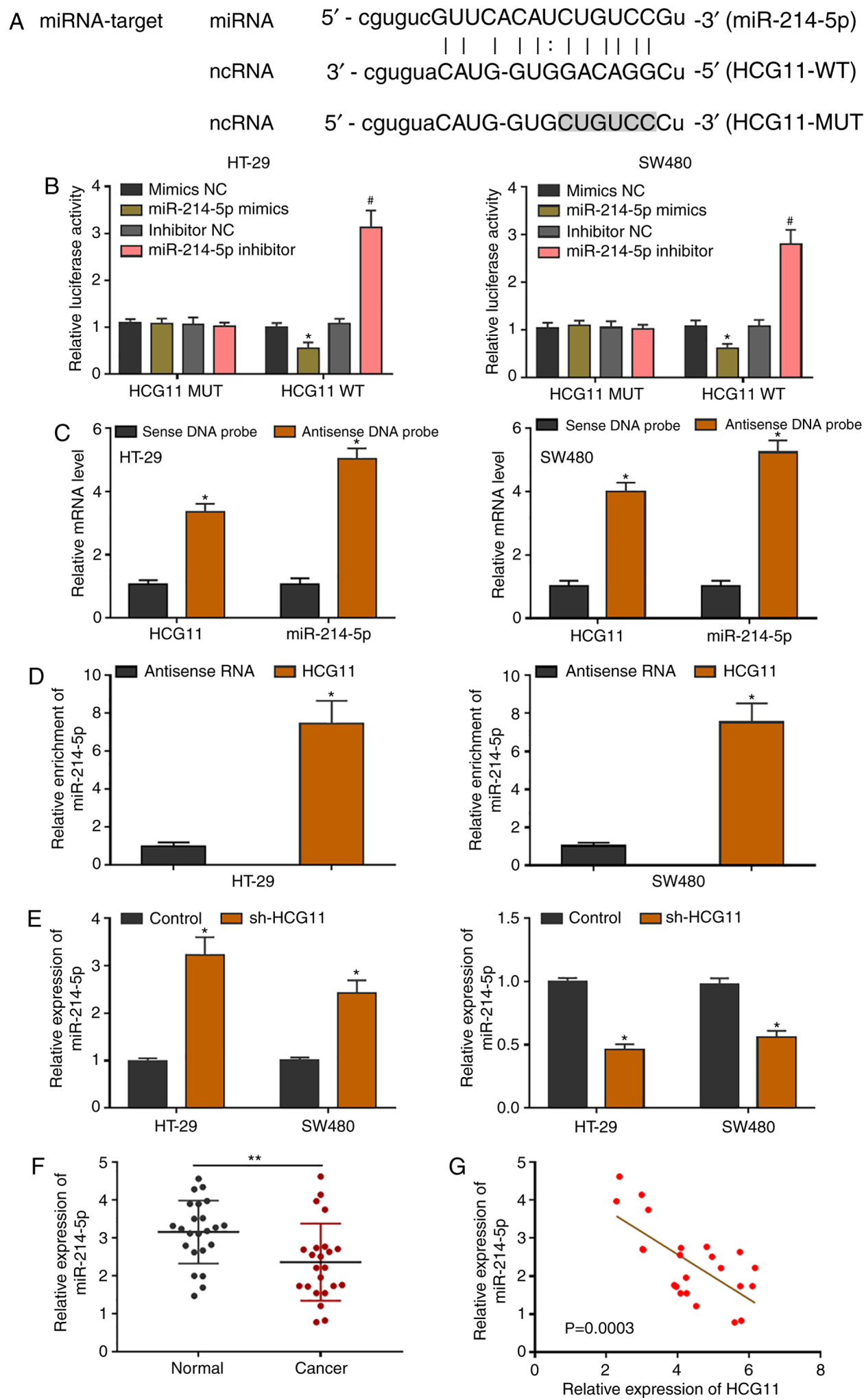

Figure 4. miR-214-5p is a target gene of HCG11. (A) Mutation sequence of binding sites between the 3' untranslated region of HCG11 and miR-214-5p, as predicted using the starBase database. (B) Dual-luciferase reporter assay of HCG11 binding to miR-214-5p in colorectal carcinoma cells. (C) In RNAs pulled down by a biotin-labeled HCG11 DNA probe, rich expression of miR-214-5p was observed, suggesting binding in colorectal carcinoma cells. (D) Through co-incubation of the full-length, biotin-labeled HCG11 sequence and CRC cell lysis solution, rich expression of miR-214-5p in the extracted RNA was shown by RT-qPCR. (E) miR-214-5p presented with the opposite changes with knockdown or overexpression of HCG11 in colorectal carcinoma cells. (F) RT-qPCR analysis of miR-214-5p downregulation in colorectal cancer tissues. (G) Negative correlation between HCG11 and miR-214-5p expression in clinical tumor samples was determined by Pearson's analysis. ${ }^{*} \mathrm{P}<0.05$ and ${ }^{\#} \mathrm{P}<0.05$ vs. control or normal tissues. HCG11, HLA complex group 11 ; miR, microRNA; NC, negative control; MUT, mutant; WT, wild-type; RT-q, reverse transcription-quantitative.

of HCG11. The potential binding sites are shown in Fig. 4A, and a dual-luciferase reporter assay was used to demonstrate the binding association. Binding site plasmids containing the HCG11 sequences were co-transfected with miR-214-5p 
mimics or inhibitor and the corresponding negative controls, and luciferase activity was then detected. The results showed a binding association between HCG11 and miR-214-5p (Fig. 4B). An RNA pull-down experiment was conducted to analyze gene-binding and complex-formation ability. In HT-29 and SW480 cells, high expression of miR-214-5p was observed in HCG11-anchored RNA, which further confirmed their binding state in CRC cells (Fig. 4C and D). CRC cells were selected for the targeted knockdown of HCG11 to detect changes in miR-214-5p expression 24 h post-transfection. The upregulation of miR-214-5p expression in tumor cells was observed. After HCG11-overexpression, miR-214-5p expression in tumor cells was downregulated (Fig. 4E). miR-214-5p expression was also detected in clinical tissues, and was higher in normal tissues than in CRC tissues (Fig. 4F). Pearson's correlation analysis revealed a significant negative correlation between miR-214-5p and HCG11 expression in clinical tissues (Fig. 4G). These findings indicated that miR-214-5p bound to and suppressed the expression of HCG11 in CRC, suggesting a potential mechanism of action for HCG11.

Inhibition of miR-214-5p reverses the effects of HCG11 on $C R C$ cell malignancy. A rescue experiment was performed to determine the involvement of miR-214-5p in the regulatory effects of HCG11 on CRC. It was demonstrated that under normal conditions, or CRC cell culture under chemotherapy, the HCG11-induced inhibition of cellular proliferation and migration were recovered by $\mathrm{m}$ iR-214-5p-knockdown. RT-qPCR demonstrated that the miR-214-5p inhibitor and mimics resulted in miR-214-5p expression in HT-29 and SW480 cells (Fig. 5A). Then, CCK-8 assays indicated that the proliferation of HT-29 and SW480 cells was reactivated by inhibiting miR-214-5p expression (Fig. 5B and C). Furthermore, EdU experiments indicated that miR-214-5p-knockdown restored the proliferative activity of tumor cells (Fig. 5D). Similarly, Transwell assays showed that miR-214-5p-knockdown rescued the migration ability of tumor cells (Fig. 5E and F). The colony formation assay also indicated that miR-214-5p-knockdown improved the clonal propagation of tumor cells (Fig. 5G). Compared with si-HCG11-transfected CRC cells, CRC cells co-transfected with si-HCG11+miR-214-5p inhibitor showed drug resistance to cisplatin. The IC50 value of co-transfected CRC cells was increased accordingly (Fig. 5H). Mechanistically, the EMT pathway in CRC cells was inhibited by si-HCG11 transfection, but was restored by si-HCG11+miR-214-5p inhibitor co-transfection (Fig. 5I). These results indicate that in CRC cells, miR-214-5p is involved in the regulatory carcinogenic pathway.

SOX4 is a target of the HCG11/miR-214-5p circuit and involved in EMT pathway mediation. It is well known that miRNAs widely regulate cellular mRNAs, and exert corresponding regulatory roles. In CRC, potential mRNA targets of miR-214-5p were predicted, and SOX4 was found to be a candidate target gene (Fig. 6A). Analysis of TCGA database revealed that SOX4 expression was higher in the CRC cohort than in normal colon tissues (Fig. 6B). Based on the starBase prediction results, the binding sites of miR-214-5p and SOX4 are shown in Fig. 6C. A dual-luciferase reporter gene assay and gene interference assay were used to verify the binding relationship between miR-214-5p and its target gene SOX4 in CRC, and demonstrated that miR-214-5p suppressed SOX4 expression by binding to its 3' UTR region (Fig. 6D and E). To verify the association between HCG11 and SOX4, SOX4 expression was detected in CRC patient specimens. The results of correlation analysis revealed a positive relationship between HCG11 and SOX4 (Fig. 6F). Furthermore, RT-qPCR revealed that the mRNA level of SOX4 was influenced by HCG11 in CRC cells (Fig. 6G). The transfection efficiency of si-SOX4 as verified by RT-qPCR (Fig. 6H), which also revealed that the $\mathrm{HCG} 11 / \mathrm{miR}-214-5 \mathrm{p}$ loop regulates the mRNA level of SOX4 in tumor cells (Fig. 6I). In addition, western blotting showed that SOX4 protein expression was affected by the HCG11/miR-214-5p axis in CRC cells (Fig. 6J). Following transfection with the miR-214-5p inhibitor, the protein level of E-cadherin was notably reduced, whereas the protein level of vimentin was increased. However, this process was reversed by co-transfection with si-SOX4 and the miR-214-5p inhibitor (Fig. 6K). Collectively, these results identified SOX4 as a target of the HCG11/miR-214-5p circuit, and confirmed that SOX4 mediates the EMT pathway in SW480 and HT-29 cells.

\section{Discussion}

The drug resistance of tumors has been widely studied, and the resistance of most tumor types to treatment frequently results in treatment failure and poor prognosis $(24,25)$. Therefore, it is necessary to investigate the genetic features of drug resistance in tumors to further understand the mechanisms of tumor progression, and to identify novel therapeutic targets. The chemotherapeutic resistance of CRC is a treatment challenge associated with patient prognosis and recurrence $(26,27)$. HCG11 was found to be highly expressed in CRC tissues and to act as a marker for poor prognosis in clinical assessments. It is also worth noting that HCG11 accelerates the progression of various human tumors, and is also involved in the induction of tumor proliferation, migration and other malignant abilities $(14,28)$. Also, the addition of chemotherapeutic drugs to the CRC cell culture medium considerably downregulated HCG11 gene expression, suggesting a potential association between HCG11 and CRC chemotherapy, albeit with no reports of HCG11 involvement in any chemotherapy. Our subsequent experiments demonstrated that in CRC, HCG11 promoted the proliferation and migration of tumor cells, induced the resistance of CRC cells to chemotherapy, and improved the IC50 value of chemotherapy compounds towards CRC cells. HCG11 was also found to be involved in mediating the chemotherapeutic sensitivity of CRC cells; however, its mechanism remains unclear. The results of the present study indicated that high expression of HCG11 enhanced the chemotherapy resistance and maintained the malignant activity of CRC cells, including proliferation and metastasis. These results imply that HCG11 is closely involved in chemotherapeutic resistance and predicts poor prognosis in patients with CRC.

lncRNAs are able to sponge their miRNA targets in cells, thus limiting gene expression by exerting post-transcriptional regulatory functions $(29,30)$. As predicted using bioinformatics techniques, miR-214-5p is a potential molecular target of HCG11, and dual-luciferase and RNA pull-down experiments 
A
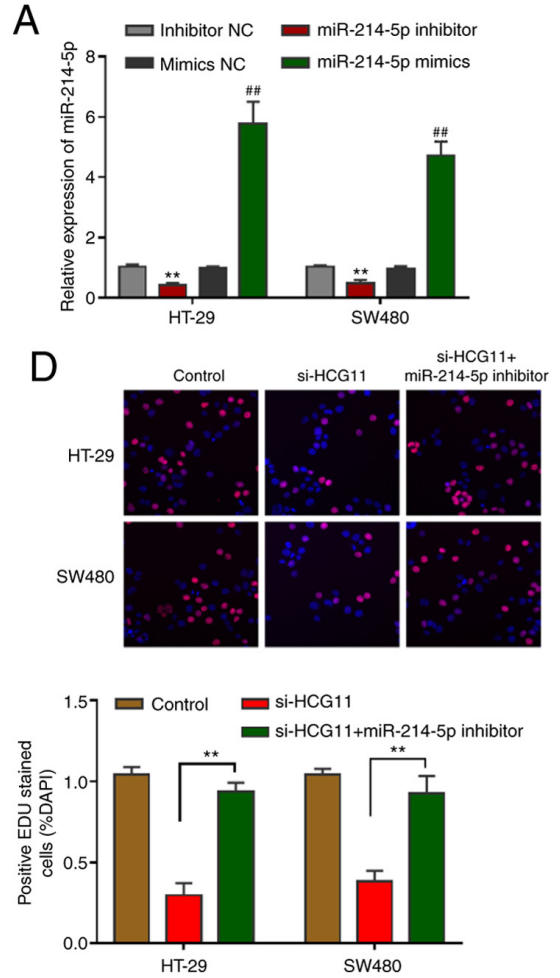

G

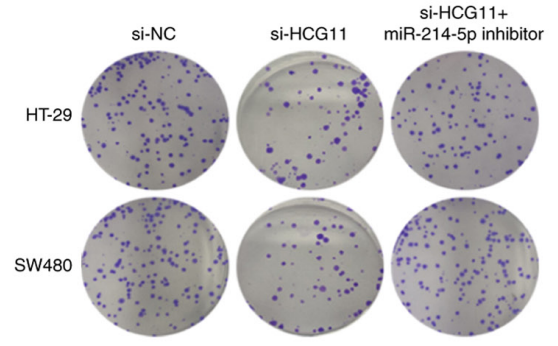

I

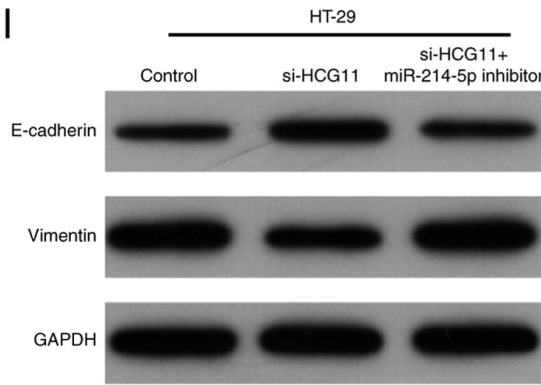

B

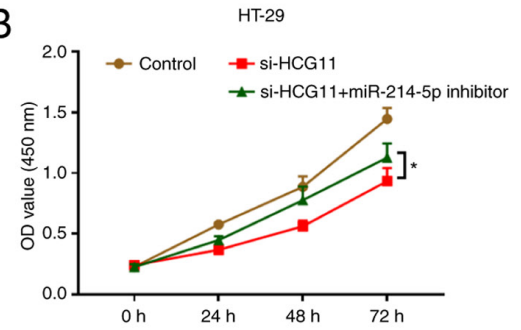

E
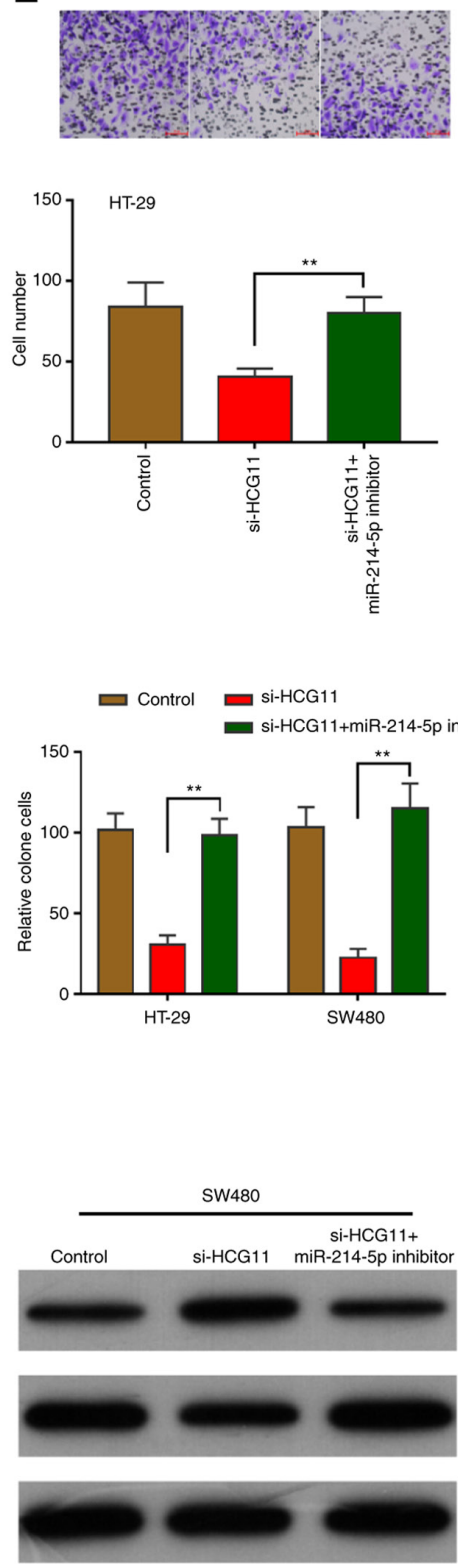

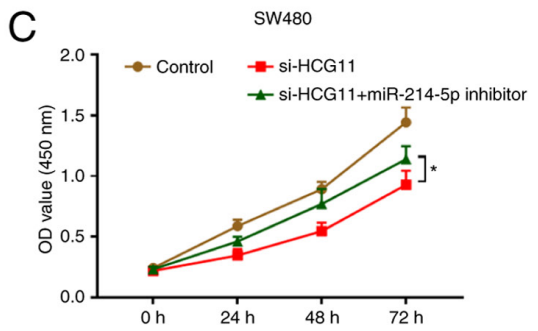

F
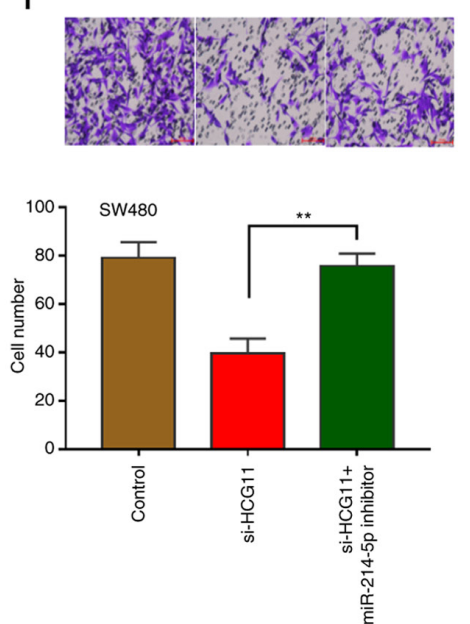

$\mathrm{H}$
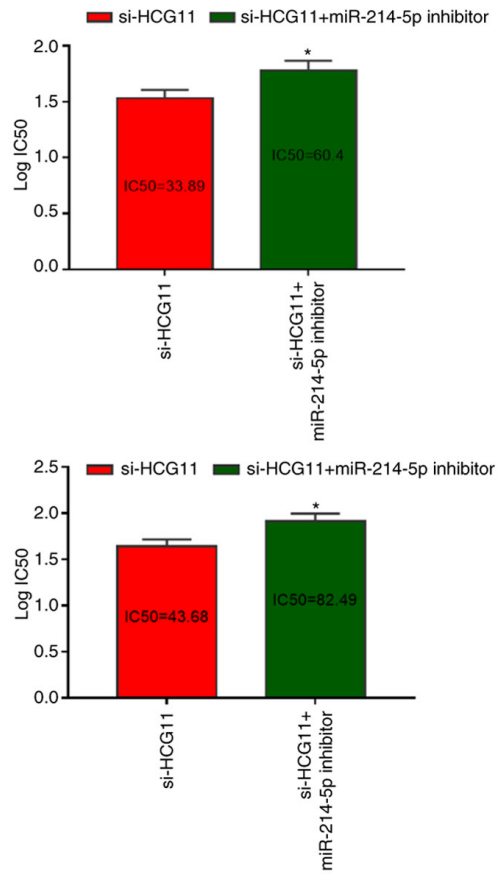

Figure 5. miR-214-5p reverses the effects of HCG11 on CRC cell malignancy. (A) Transfection efficiency of miR-214-5p mimics and miR-214-5p inhibitor as verified by reverse transcription-quantitative PCR. Partial recovery of (B) HT-29 and (C) SW480 colorectal carcinoma cell proliferation following co-transfection of si-HCG11 and miR-214-5p inhibitor by Cell Counting Kit-8. (D) Partial recovery of cellular proliferation following co-transfection of si-HCG11 and miR-214-5p inhibitor via EdU staining. The recovery of (E) HT-29 and (F) SW480 colorectal carcinoma cell migration following co-transfection of si-HCG11 and miR-214-5p inhibitor via Transwell. (G) Recovery of cell proliferation after co-transfection of si-HCG11 and miR-214-5p inhibitor shown using colony formation assays. $(\mathrm{H})$ Higher sensitivity of $\mathrm{CRC}$ cells to cisplatin and higher restoration of $\mathrm{IC}_{50}$ values following co-transfection of si-HCG11 and miR-214-5p inhibitor. (I) Epithelial-mesenchymal transition pathway restoration by si-HCG11+miR-214-5p inhibitor co-transfection. ${ }^{*} \mathrm{P}<0.05$ and ${ }^{* *} \mathrm{P}<0.01$ vs. control. miR, microRNA; NC, negative control; HCG11, HLA complex group 11; Si, small interfering (RNA); EdU, 5-ethynyl-2'-deoxyuridine.

verified the correlation between binding and mutual regulation in CRC cells. In previous studies, miR-214-5p was found to regulate constituents of intracellular signaling pathways, including the jagged 1 gene and EMT-related genes in pancreatic cancer (31), as well as regulating dihydropyrimidinase-related protein 5 expression in prostatic cancer (32), 
A

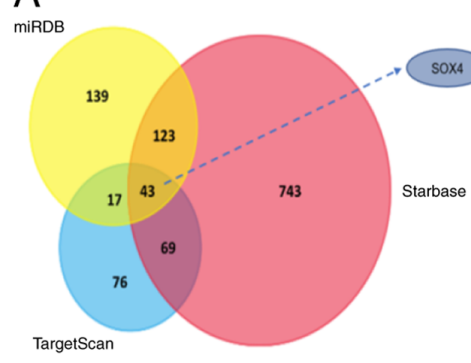

B

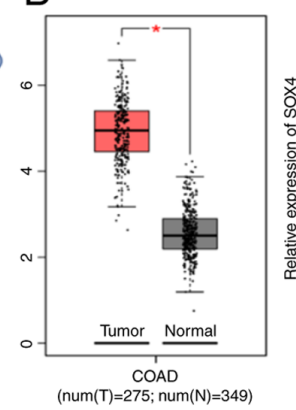

C

miRNA-target miRNA $3^{\prime}$ - cgugucguucacauCUGUCCGu-5' (miR-214-5p)

|||||| $\mid$

mRNA 5 ' - guuuaauauaacauGACAGGCa -3' (SOX4-WT)

mRNA $\quad 5^{\prime}$ - guuuaauauaacauCUGUCCGa -3' (SOX4-MUT)
D

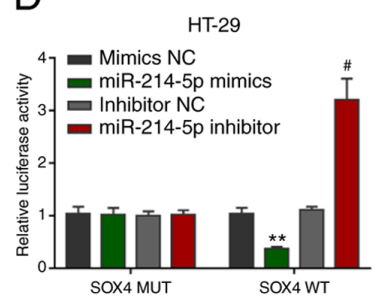

$\mathrm{F}$

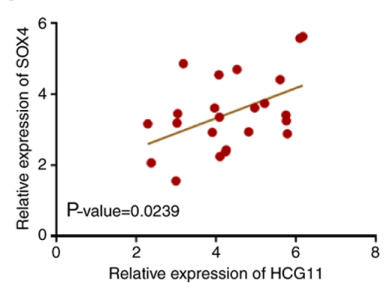

SW 480

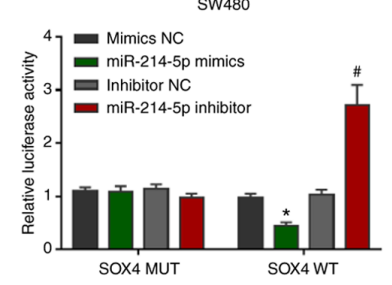

G

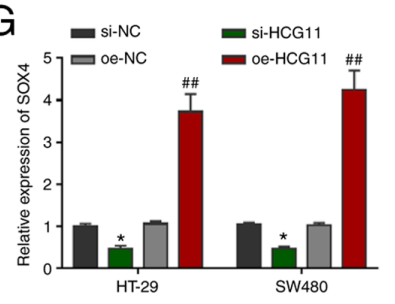

E

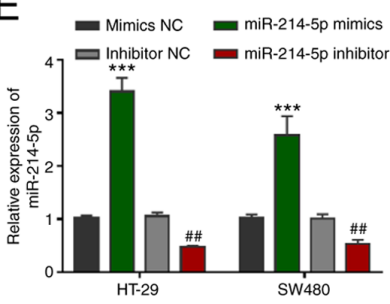

$\mathrm{H}$
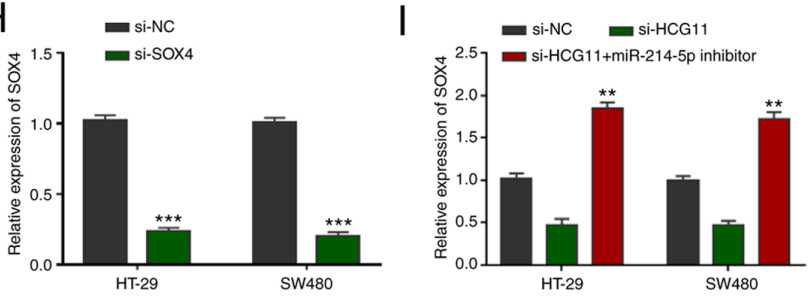

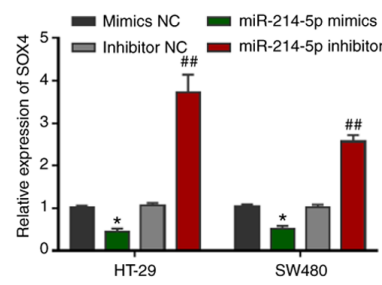

SW480

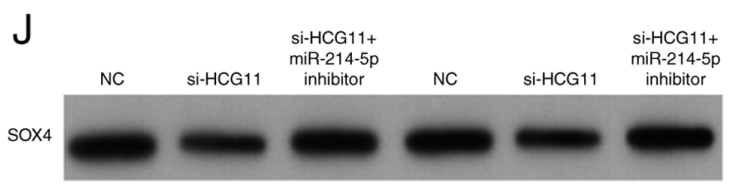

GAPDH

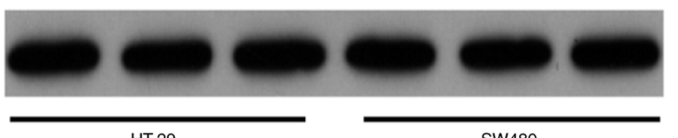

HT-29

SW480
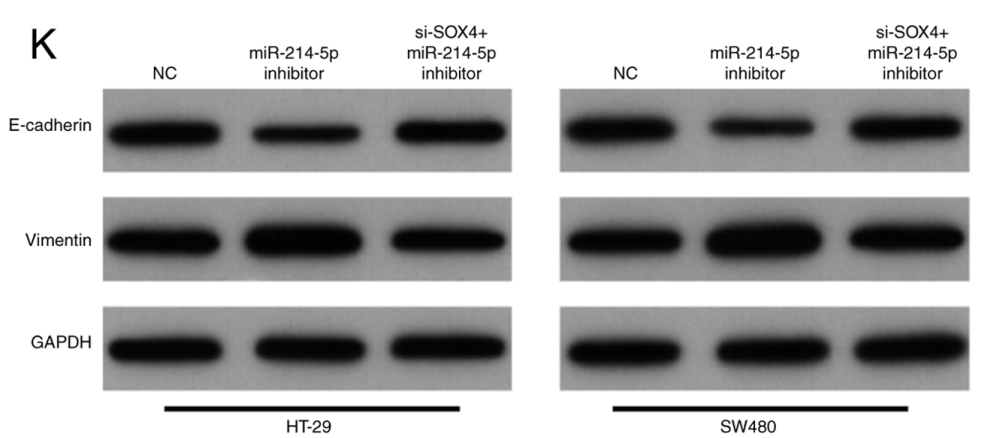

Figure 6. SOX4 is a target of the HCG11/miR-214-5p axis and is involved in EMT. (A) SOX4 was found to be a downstream target gene of miR-214-5p, as predicted by Venn diagram screening. (B) High expression of SOX4 in colorectal carcinoma tissues as shown by The Cancer Genome Atlas database analysis (C) Predicted binding sites between SOX4 mRNA and miR-214-5p. (D) Mutual binding between SOX4 and miR-214-5p was confirmed by the dual-luciferase reporter assays. (E) Interference of miR-214-5p expression in colorectal carcinoma cells indirectly negatively regulated the mRNA level of SOX4. (F) HCG11 expression was positively correlated with that of SOX4 in colorectal carcinoma tissue samples. (G) Interference of HCG11 expression in colorectal carcinoma cells regulated the mRNA level of SOX4 in tumor cells. (H) Transfection efficiency of si-SOX4 as verified by RT-qPCR. (I) RT-qPCR results showed that the HCG11/miR-214-5p loop regulated SOX4 mRNA levels in tumor cells. (J) Western blot results revealed that the HCG11/miR-214-5p loop regulated the protein level of SOX4 in tumor cells. (K) Western blot results revealed that miR-214-5p regulated the level of SOX4-mediated epithelial-mesenchymal transition. ${ }^{*} \mathrm{P}<0.05,{ }^{* *} \mathrm{P}<0.01$ and ${ }^{* * * *} \mathrm{P}<0.001$, and ${ }^{\#} \mathrm{P}<0.05$ and ${ }^{\# \#} \mathrm{P}<0.01$ vs. the corresponding control. miR, microRNA; NC, negative control; HCG11, HLA complex group 11; $\mathrm{Si}$, small interfering; oe, overexpression; RT-q, reverse transcription-quantitative; MUT, mutant; WT, wild-type; COAD, colon adenocarcinoma.

and the expression of the target gene of rho-associated protein kinase 1 by direct binding in bone sarcoma (33). However, to the best of our knowledge, no study has demonstrated tumor protection of CRC cells. Therefore, an intracellular 
functional reverse experiment was performed, which revealed that miR-214-5p markedly suppressed the carcinogenesis of HCG11-induced CRC. Similarly, miR-214-5p-overexpression notably recovered the inhibitory effects of HCG11 on the chemotherapeutic sensitivity of CRC. The results of the present study indicate the important roles of HCG11/miR-214-5p in $\mathrm{CRC}$ as a new cyclic regulatory mechanism.

As a target gene of miR-214-5p, SOX4 has previously been identified as a key oncogene in various different tumors (34). The primary protein functions regulated by SOX 4 include the mediation of tumorigenesis and involvement in various EMT-associated signaling pathways (35). The SOX family of protein are closely associated with tumor progression under the tight regulation of miRNAs and lncRNAs (36). SOX4 is associated with the prognosis and grading of CRC, and increased SOX4 expression has been identified as a marker of poor prognosis (37). Data mining analysis of TCGA revealed higher expression levels of SOX4 in CRC than in normal cells. EMT pathways regulated by SOX4 are key signaling pathways that mediate the chemotherapeutic resistance of tumor cells $(38,39)$. The present study revealed that miR-214-5p binds to and negatively regulates $\mathrm{SOX} 4$ expression. Furthermore, high levels of SOX4 expression inhibited EMT in CRC cells, while co-transfection with miR-214-5p suppressed the EMT to protect cells from chemotherapeutic drug resistance. This process is known as the competing endogenous RNA mechanism, in which HCG11 promotes tumor progression in CRC cells. In summary, the results of the present study demonstrated that IncRNA HCG11 mediated the chemotherapy resistance of CRC cells, and elucidated the potential mechanisms of action (in the form of an IncRNA-miRNA-mRNA axis) to further explain the causes of chemotherapy resistance in CRC.

In conclusion, the present study demonstrated that lncRNA HCG11 is significantly upregulated in CRC, and associated with chemotherapeutic resistance of and poor prognosis. HCG11-knockdown markedly reduced the proliferation and migration of CRC cells and increased the sensitivity of tumor cells to chemotherapy. However, HCG11 overexpression enhanced the chemotherapy resistance of CRC cells. Therefore, these data indicate that HCG11 might act as an oncogene in CRC, similar to previously reported tumor studies. Further studies identified miR-214-5p as a target gene of HCG11, which mediates EMT regulation in tumor cells, and is a key downstream molecule for HCG11-associated carcinogenicity. However, further studies are required to evaluate the role of HCG11 as a therapeutic target in CRC.

\section{Acknowledgements}

Not applicable.

\section{Funding}

No funding was received.

\section{Availability of data and materials}

All data generated or analyzed during this study are included in this published article.

\section{Authors' contributions}

JP and YM conceived the study idea; JX and YM participated in the design and implementation of the experiments; JX and JZ analysed the final data and JX wrote the manuscript. All authors have read and approved the manuscript, and agree to be responsible for all aspects of the study to ensure the accuracy or completeness of any part of the work is properly investigated and resolved.

\section{Ethics approval and consent to participate}

The present study was approved by the Ethics Committees of the First Affiliated Hospital of Yangtze University. All enrolled patients provided written informed consent.

\section{Patient consent for publication}

Not applicable.

\section{Competing interests}

The authors declare that they have no competing interests.

\section{References}

1. Gu L, Xu Y, Xu W, Li M, Su H, Li C and Liu Z: The exosome secretion inhibitor neticonazole suppresses intestinal dysbacteriosis-induced tumorigenesis of colorectal cancer. Invest New Drugs 38: 221-228, 2020.

2. Fidler MM and Bray F: Global cancer inequalities. Front Oncol 8: 293,2018

3. Hong J, Lu H, Meng X, Ryu JH, Hara Y and Yang CS: Stability, cellular uptake, biotransformation, and efflux of tea polyphenol (-)-epigallocatechin-3-gallate in HT-29 human colon adenocarcinoma cells. Cancer Res 62: 7241-7246, 2002.

4. Araghi M, Soerjomataram I, Bardot A, Ferlay J, Cabasag CJ, Morrison DS, De P, Tervonen H, Walsh PM, Bucher O, et al: Changes in colorectal cancer incidence in seven high-income countries: A population-based study. Lancet Gastroenterol Hepatol 4: 511-518, 2019.

5. Tsukuda K, Tanino M, Soga H, Shimizu N and Shimizu K: A novel activating mutation of the K-ras gene in human primary colon adenocarcinoma. Biochem Biophys Res Commun 278: $653-658,2000$

6. Wapinski $\mathrm{O}$ and Chang HY: Long noncoding RNAs and human disease. Trends Cell Biol 21: 354-361, 2011.

7. Wang KC and Chang HY: Molecular mechanisms of long noncoding RNAs. Mol Cell 43: 904-914, 2011.

8. Mercer TR, Dinger ME and Mattick JS: Long non-coding RNAs: Insights into functions. Nat Rev Genet 10: 155-159, 2009.

9. Deng H, Wang JM, Li M, Tang R, Tang K, Su Y, Hou Y and Zhang J: Long non-coding RNAs: New biomarkers for prognosis and diagnosis of colon cancer. Tumour Biol: Jun 23, 2017 (Epub ahead of print).

10. Cheng B, Rong A,Zhou Q and Li W: LncRNA LINC00662 promotes colon cancer tumor growth and metastasis by competitively binding with miR-340-5p to regulate CLDN8/IL22 co-expression and activating ERK signaling pathway. J Exp Clin Cancer Res 39: 5, 2020.

11. Xu T, Wu K, Zhang L, Zheng S, Wang X, Zuo H, Wu X, Tao G, Jiang B and Zhang L: Long non-coding RNA LINC00858 exerts a tumor-promoting role in colon cancer via HNF4 $\alpha$ and WNK2 regulation. Cell Oncol (Dordr) 43: 297-310, 2020.

12. Zhang H, Huang H, Xu X, Wang H, Wang J, Yao Z, Xu X, Wu Q and Xu F: LncRNA HCG11 promotes proliferation and migration in gastric cancer via targeting miR-1276/CTNNB1 and activating Wnt signaling pathway. Cancer Cell Int 19: 350, 2019.

13. Chen Y, Bao C, Zhang X, Lin X, Huang H and Wang Z: Long non-coding RNA HCG11 modulates glioma progression through cooperating with miR-496/CPEB3 axis. Cell Prolif 52: e12615, 2019. 
14. Wang YC, He WY, Dong CH, Pei L and Ma YL: lncRNA HCG11 regulates cell progression by targeting miR-543 and regulating AKT/mTOR pathway in prostate cancer. Cell Biol Int 43: $1453-1462,2019$

15. Greenburg G and Hay ED: Epithelia suspended in collagen gels can lose polarity and express characteristics of migrating mesenchymal cells. J Cell Biol 95: 333-339, 1982.

16. Skrypek N, Goossens S, De Smedt E, Vandamme N and Berx G: Epithelial-to-mesenchymal transition: Epigenetic reprogramming driving cellular plasticity. Trends Genet 33: 943-959, 2017.

17. Loric S, Paradis V, Gala JL, Berteau P, Bedossa P, Benoit G and Eschwège P: Abnormal E-cadherin expression and prostate cell blood dissemination as markers of biological recurrence in cancer. Eur J Cancer 37: 1475-1481, 2001.

18. Sato M, Shames DS and Hasegawa Y: Emerging evidence of epithelial-to-mesenchymal transition in lung carcinogenesis. Respirology 17: 1048-1059, 2012.

19. Qiu E, Gao Y,Zhang B, Xia T, Zhang Z and Shang G: Upregulation of cell division cycle 20 in cisplatin resistance-induced epithelial-mesenchymal transition in osteosarcoma cells. Am J Transl Res 12: 1309-1318, 2020.

20. Ye Y, Gu J, Liu P, Wang H, Jiang L, Lei T, Yu S, Han G and Wang Z: Long non-coding RNA SPRY4-IT1 reverses cisplatin resistance by downregulating MPZL-1 via suppressing EMT in NSCLC. OncoTargets Ther 13: 2783-2793, 2020

21. Acikgoz E, Tatar C and Oktem G: Triptolide inhibits $\mathrm{CD} 133^{+} / \mathrm{CD} 44^{+}$colon cancer stem cell growth and migration through triggering apoptosis and represses epithelial-mesenchymal transition via downregulating expressions of snail, slug, and twist. J Cell Biochem 121: 3313-3324, 2020.

22. Livak KJ and Schmittgen TD: Analysis of relative gene expression data using real-time quantitative PCR and the 2(-Delta Delta C(T)) method. Methods 25: 402-408, 2001.

23. Tang Z, Li C, Kang B, Gao G, Li C and Zhang Z: GEPIA: A web server for cancer and normal gene expression profiling and interactive analyses. Nucleic Acids Res 45: W98-W102, 2017

24. Eberhardt W, Nasrullah $U$ and Haeussler K: Inhibition of Caspase- 2 translation by the mRNA binding protein $\mathrm{HuR}$ : A novel path of therapy resistance in colon carcinoma Cells? Cells 8: E797, 2019

25. Paldino E, Tesori V, Casalbore P, Gasbarrini A and Puglisi MA: Tumor initiating cells and chemoresistance: Which is the best strategy to target colon cancer stem cells? BioMed Res Int 2014: 859871, 2014.

26. Kozovska Z, Gabrisova V and Kucerova L: Colon cancer: Cancer stem cells markers, drug resistance and treatment. Biomed Pharmacother 68: 911-916, 2014.
27. Uppada SB, Gowrikumar S, Ahmad R, Kumar B, Szeglin B, Chen X, Smith JJ, Batra SK, Singh AB and Dhawan P: MASTL induces colon cancer progression and chemoresistance by promoting Wnt/ $\beta$-catenin signaling. Mol Cancer 17: 111, 2018.

28. Frankiewicz $\mathrm{Z}$ and Leski J: Adaptive fiducial point detector for ECG stress testing systems. Int J Biomed Comput 28: 127-135, 1991.

29. Qi X, Zhang DH, Wu N, Xiao JH, Wang X and Ma W: ceRNA in cancer: Possible functions and clinical implications. J Med Genet 52: 710-718, 2015.

30. Chan JJ and Tay Y: Noncoding RNA: RNA Regulatory Networks in Cancer. Int J Mol Sci 19: E1310, 2018.

31. Cao TH, Ling X, Chen C, Tang W, Hu DM and Yin GJ: Role of miR-214-5p in the migration and invasion of pancreatic cancer cells. Eur Rev Med Pharmacol Sci 22: 7214-7221, 2018

32. Zheng C, Guo K, Chen B, Wen Y and Xu Y: miR-214-5p inhibits human prostate cancer proliferation and migration through regulating CRMP5. Cancer Biomark 26: 193-202, 2019.

33. Zhang M, Wang D, Zhu $\mathrm{T}$ and Yin R: miR-214-5p targets ROCK1 and suppresses proliferation and invasion of human osteosarcoma cells. Oncol Res 25: 75-81, 2017.

34. Ding L, Zhao Y, Dang S, Wang Y, Li X, Yu X, Li Z, Wei J, Liu M and Li G: Circular RNA circ-DONSON facilitates gastric cancer growth and invasion via NURF complex dependent activation of transcription factor SOX4. Mol Cancer 18: 45, 2019.

35. Hanieh H, Ahmed EA, Vishnubalaj R and Alajez NM: SOX4: Epigenetic regulation and role in tumorigenesis. Semin Cancer Biol 67: 91-104, 2020.

36. Grimm D, Bauer J, Wise P, Krüger M, Simonsen U, Wehland M, Infanger $\mathrm{M}$ and Corydon TJ: The role of SOX family members in solid tumours and metastasis. Semin Cancer Biol 67: 122-153, 2020.

37. Lin CM, Fang CL, Hseu YC, Chen CL, Wang JW, Hsu SL, Tu MD, Hung ST, Tai C, Uen YH, et al; Clinical and prognostic implications of transcription factor SOX4 in patients with colon cancer: Clinical and prognostic implications of transcription factor SOX4 in patients with colon cancer. PLoS One 8: e67128, 2013.

38. Shibue T and Weinberg RA: EMT, CSCs, and drug resistance: The mechanistic link and clinical implications. Nat Rev Clin Oncol 14: 611-629, 2017.

39. Du B and Shim JS: Targeting epithelial-mesenchymal transition (EMT) to overcome drug resistance in cancer. Molecules 21: $\mathrm{E} 965,2016$

This work is licensed under a Creative Commons Attribution-NonCommercial-NoDerivatives 4.0 International (CC BY-NC-ND 4.0) License. 